\title{
CYCLOTOMY AND DELTA UNITS
}

\author{
ANDREW J. LAZARUS
}

To the memory of Derrick Henry Lehmer

\begin{abstract}
In this paper we examine cyclic cubic, quartic, and quintic number fields of prime conductor $p$ containing units that bear a special relationship to the classical Gaussian periods: $\eta_{j}-\eta_{j+1}+c$ is a unit for periods $\eta_{j}$ and $c \in \mathbb{Z}$.
\end{abstract}

\section{INTRODUCTION}

In [10], Emma Lehmer discovered that certain well-known families of cubic and quartic fields contained translation units, where a translation unit $\theta$ differs from a Gaussian period $\eta$ by a rational integer. She then presented a family of quintic fields with the same property. Schoof and Washington [11] proved the converse of Lehmer's results for cubic fields and those quartic fields in which all units have norm +1 .

Later D. H. and Emma Lehmer became interested in a cyclotomy where the Gaussian period $\eta$ was replaced by the difference $\delta_{j}$ of two periods $\eta_{j}-\eta_{j+1}$. We will show that the fields with analogously-defined delta units are, in the cubic and quartic cases, the same as those already known. In Lehmer's quintic case the situation is more complicated because the ordering of the $\eta$ 's is not unique. The Lehmers observed without proof in [9] that only half of the primitive roots $\bmod p$ induce an ordering of the $\eta$ 's which give a delta unit in the quintic field of conductor $p$. We investigate this phenomenon.

\section{Definitions}

The cyclotomic classes of degree $e$ and prime conductor $p=e f+1$ are

$$
\mathscr{C}_{j}=\left\{g^{e \nu+j} \bmod p: \nu=0, \ldots, f-1\right\}, \quad j=0, \ldots, e-1,
$$

where $g$ is any primitive root $\bmod p$. Here, $\mathscr{C}_{0}$ contains the $e$ th-power residues, but the ordering of the other classes depends upon the choice of $g$. The Gaussian periods $\eta$ are defined by

$$
\eta_{j}=\sum_{\nu \in \mathcal{F}_{j}} \zeta_{p}^{\nu}, \quad j=0, \ldots, e-1,
$$

Received by the editor June 26, 1992.

1991 Mathematics Subject Classification. Primary 11R27, 11R16, 11R20; Secondary 11L99.

Key words and phrases. Units, cyclotomy, Gaussian periods. 
where $\zeta_{p}=\exp (2 \pi i / p)$. The Lagrange resolvent $\tau$, sometimes called a Gauss sum, of a character $\chi$ of order $e$ (e.g., $\chi$ is a complex-valued $e$ th-power residue symbol) is

$$
\tau(\chi)=\sum_{j=0}^{p-1} \chi(j) \zeta_{p}^{j}
$$

When $\chi$ is taken to be the character defined by $\chi(g)=\zeta_{e}$, the well-known fundamental relations between Gaussian periods and Lagrange resolvents are given by

$$
\tau\left(\chi^{j}\right)=\sum_{k=0}^{e-1} \zeta_{e}^{j k} \eta_{k}, \quad \eta_{k}=e^{-1} \sum_{j=0}^{e-1} \zeta_{e}^{-j k} \tau\left(\chi^{j}\right) .
$$

The delta cyclotomy is defined by

$$
\delta_{j}=\eta_{j}-\eta_{j+1}
$$

Here and throughout, indices of $\eta$ and $\delta$ should be understood $\bmod e$; when omitted, we mean to refer to any $\eta$ or $\delta$ 's. The different orderings of the $\eta$ 's induce different values of the $\delta$ 's.

A unit $\theta$ such that $\theta=\eta+c$ for some $c \in \mathbb{Z}$ is called a translation unit. If $\theta=\delta+c$ for some $\delta$ defined by (2.3), then $\theta$ is a generalized delta unit; if $\theta=\delta \pm 1$, then $\theta$ is a delta unit.

\section{CUBiC FIELDS}

Since the conductor $p \equiv 1 \bmod 6$, we have the well-known decomposition

$$
4 p=L^{2}+27 M^{2}, \quad L \equiv 1 \bmod 3, M>0 .
$$

We may assume that $g$ is chosen such that [5, Proposition 1]

$$
g^{(p-1) / 3} \equiv(L+9 M) /(L-9 M) \quad \bmod p .
$$

Theorem 1. If $K$ is a cyclic cubic field of prime conductor $p$, the following are equivalent:

(i) $M=1$, so $K$ is a simplest cubic as defined by Shanks [12].

(ii) $K$ has a translation unit.

(iii) $K$ has a delta unit.

(iv) $K$ has a generalized delta unit.

Proof. (i) $\Rightarrow((\mathrm{ii}) \&$ (iii)): Shanks showed that the polynomials

$$
Y^{3}-\frac{L-3}{2} Y^{2}-\frac{L+3}{2} Y-1=\prod_{j=0}^{2}\left(Y-\theta_{j}\right)
$$

generate the cubic fields with $M=1$. Emma Lehmer showed that $\eta+(L-1) / 6$ is one of the units $\theta$ [10]. The Lehmers showed in [9] that if $M=1$, then $\delta-1$ is a unit.

(iii) $\Rightarrow$ (iv): Trivial.

(ii) $\Rightarrow(\mathrm{i})$ : This is shown in [11]. 
(iv) $\Rightarrow(\mathrm{i})$ : We can find the minimal polynomial $\operatorname{Irr}_{\mathbb{Q}} \delta$ from the definition (2.3) and the cyclotomic numbers of order 3 . These are defined (for fixed g) by

$$
(h, k)=\#\left\{\nu \in(\mathbb{Z} / p \mathbb{Z})^{*}: \nu \in \mathscr{C}_{h}^{(g)}, \nu+1 \in \mathscr{C}_{k}^{(g)}\right\} .
$$

There are a number of well-known general formulas satisfied by the cyclotomic numbers (see, e.g., $[1,13])$, including

$$
\begin{gathered}
\eta_{a} \eta_{a+k}=\epsilon^{(k)} f+\sum_{h=0}^{e-1}(h, k) \eta_{a+h}, \\
\epsilon^{(k)}= \begin{cases}1, & k=0, f \text { even, or } k=e / 2, f \text { odd }, \\
0, & \text { otherwise. }\end{cases}
\end{gathered}
$$

The cyclotomic numbers for $e=3$ were determined in principle by Gauss. For $g$ normalized by (3.1), we have [5, Proposition 1 , misprint corrected]

$$
\begin{gathered}
(00)=(p-8+L) / 9 \\
(11)=(20)=(02)=(2 p-4-L-9 M) / 18 \\
(01)=(10)=(22)=(2 p-4-L+9 M) / 18 \\
(12)=(21)=(p+1+L) / 9 .
\end{gathered}
$$

It is now a routine computation to find that

$$
\operatorname{Irr}_{\mathbb{Q}} \delta=X^{3}-p X+M p
$$

We are therefore looking to solve

$$
N_{\mathbb{Q}}^{K}(\delta+c)=c^{3}-p(c+M)= \pm 1
$$

If $c=-1$, it is immediate that the only solution is $M=1$ and a norm of -1 . If $c=1$, there are no units. First, $p=7$ (where $M=1$ ) can be checked as a special case. For $p>7$, we have $1-p+M<1+2 \sqrt{p}-p<-1$. This shows (iii) $\Rightarrow$ (i).

Generalized delta units of norm +1 would be, from (3.4), solutions to

$$
(c-1)\left(c^{2}+c+1\right)=p(c+M) \text {. }
$$

Since $p$ is prime, it divides one of the factors on the left. If

$$
d p=c^{2}+c+1
$$

then

$$
d(c-1)=c+M
$$

Isolating $M$, gives

$$
M=c d-c-d=(c-1)(d-1)-1 .
$$

From (3.5) and $p>0$ we have $d>0$. Combining this with (3.7) and $M>0$ forces $d \geq 2$ and $c \geq 2$. When $c=2$, hence $p=7$ and $M=1,(3.6)$ is not satisfied. When $c=3$, then $d=1$, a contradiction. When $c=4$, then $p=7$ and $d=3$, which gives $M=-5$, also a contradiction. Therefore, we 
may assume $c \geq 5$. Starting from (3.5), we have

$$
d p<2 c^{2} \Rightarrow L^{2}+27 M^{2}<\frac{8 c^{2}}{d} \Rightarrow M<\frac{2 \sqrt{2} c}{3 \sqrt{3 d}}<\frac{5 c}{9} .
$$

Plugging this back into (3.6), we have

$$
d(c-1)<\frac{14 c}{5} \Rightarrow d<\frac{14 c}{5(c-1)}<2
$$

(since $c \geq 5$ ), a contradiction.

Now suppose

$$
d p=c-1
$$

so

$$
M=d\left(c^{2}+c+1\right)-c .
$$

If $c=1$, we would have from (3.8) that $d=0$ and then from (3.9), $M=-1$, impossible. Moreover, $\operatorname{sgn} d=\operatorname{sgn} c$ by (3.8). When both are negative,

$$
M<d\left(c^{2}+c+1\right)+d c=d(c+1)^{2} \leq 0,
$$

a contradiction. For $c>1$, we must have that $c \geq 8$, since $p \geq 7$. Now

$$
p \leq d p<c \Rightarrow M^{2}<\frac{4 c}{27} \Rightarrow M<\sqrt{c} \text {. }
$$

Combining this with (3.9) gives the inequality $c^{2}+1<\sqrt{c}$, which never holds. Hence, there are no generalized delta units of norm +1 .

For the norm -1 case we are looking for solutions to

$$
(c+1)\left(c^{2}-c+1\right)=p(c+M) .
$$

Proceeding similarly to the positive-norm case, we first consider the possibility that $d p=c^{2}-c+1$ and $M=c d-c+d=(c+1)(d-1)+1$. As before, $d>0$. If $d=1$, we see that $M=1$ is a solution to (3.4), regardless of $c$. From now on, assume $d>1$. If $c \leq 2$, then either $p<7$ or $M<0$, which are impossible. Assume $c \geq 3$. Then

$$
d p<2 c^{2} \Rightarrow M<\frac{2 \sqrt{2} c}{3 \sqrt{3 d}} \Rightarrow d(c+1)<\frac{14 c}{5} \Rightarrow d<\frac{14 c}{9(c+1)}<2,
$$

contradicting the assumption $d \geq 2$.

The remaining case is $d p=c+1$. We have $M=d\left(c^{2}-c+1\right)-c$. If $c=-1$, then $d=0$ and $M=1$, a solution to (3.4). If $c<-1$, then $d<0$. Now

$$
M=d\left(c^{2}-c+1\right)-c<d\left(c^{2}-c+1\right)+d c<d\left(c^{2}+1\right)<0,
$$

a contradiction. It remains to check only $c \geq 0$. Immediately we get $d>0$. But then, as with $d p=c-1$, we quickly get a contradiction:

$$
p<d p<2 c \Rightarrow M<\sqrt{c} \Rightarrow c^{2}-c+1<c+\sqrt{c},
$$

and since $c \geq 6$, this, too, is impossible. 
We found all solutions to (3.4) during the proof of the theorem and summarize this result.

Corollary 3.1. All generalized delta units have norm -1 . If $M \neq 1$, there are no generalized delta units. If $M=1$, then $\delta-1$ is a unit. If, in addition, there exists $c \in \mathbb{Z}$ such that $p=c^{2}-c+1$, then $\delta+c$ and $\delta-(c-1)$ are also units.

Shanks [12] showed that when $M=1$, the group generated by -1 and any two of the units $\theta_{j}$ in (3.2) is the full unit group, and that Galois action on the units $\theta$ is given by the map $\theta \rightarrow-(\theta+1)^{-1}$. Since $\eta_{0}$ is invariant under choice of $g$, we fix $\theta_{0}$.

Proposition 3.2. The ordering of the $\eta$ induced by $\theta_{0}=\eta_{0}-(L+1) / 6$ and Shanks's map $\theta_{j+1}=-\left(\theta_{j}+1\right)^{-1}$ coincides with the ordering obtained by (2.1) and (3.1).

Proof. We find that

$$
\begin{aligned}
\left(\eta_{1}+\right. & (L-1) / 6)\left(\eta_{0}+(L+5) / 6\right) \\
& =\frac{1}{36}\left(36 \eta_{0} \eta_{1}+6 \eta_{1} L+30 \eta_{1}+6 L \eta_{0}+L^{2}+4 L-6 \eta_{0}-5\right) \\
& =\frac{1}{36}\left(4 \eta_{0} p+10 \eta_{0}-2 \eta_{0} L+4 \eta_{1} p-26 \eta_{1}-2 \eta_{1} L+4 \eta_{2} p+4 \eta_{2}+4 \eta_{2} L\right) \\
& =-1,
\end{aligned}
$$

expanding $\eta_{0} \eta_{1}$ by (3.3) and substituting in $\eta_{2}=-1-\eta_{0}-\eta_{1}$ and $p=$ $\left(L^{2}+27\right) / 4$. Therefore, $\theta_{1}=-\left(\theta_{0}+1\right)^{-1}$. Applying Galois action to both sides proves the general case.

Hasse [4] wrote elements of cyclic cubic fields as $[x, y]$, where

$$
\begin{aligned}
& {[x, y]=x-y \tau(\chi)-\overline{y \tau(\chi)} \in K,} \\
& \qquad x \in \mathbb{Z}, \quad y \in \mathbb{Q}\left[\zeta_{3}\right], \quad \chi(\cdot)=\left(\frac{\cdot}{(L+3 \sqrt{-3} M) / 2}\right)_{3} .
\end{aligned}
$$

He normalized Galois action so that $[x, y] \rightarrow\left[x, \zeta_{3} y\right]$. (Warning: Hasse used $L \equiv-1 \bmod 3$.)

Proposition 3.3. Shanks's map is the inverse of Galois action as normalized by Hasse.

Proof. It is evident from the relations (2.2) that Hasse's map takes

$$
\eta_{0}=(1+\tau(\chi)+\tau(\bar{\chi})) / 3 \rightarrow\left(1+\zeta_{3} \tau(\chi)+\zeta_{3}^{2} \tau(\bar{\chi})\right) / 3=\eta_{2},
$$

whereas the previous proposition shows that Shanks's map increments the index of $\eta$.

Delta units and the choice of $g$. Fix, for the moment, the choice of $g$. In general, redefining the periods using a generator $g^{\prime} \in \mathscr{C}_{j}^{(g)}$ yields $\eta_{\nu}^{\prime}=\eta_{\nu j}$. If $g^{\prime} \in \mathscr{C}_{-1}^{(g)}$, then $\delta_{\nu}^{\prime}=-\delta_{e-\nu}$. Therefore, in looking for delta units, $\mathscr{C}_{j}^{(g)}$ and $\mathscr{C}_{-j}^{(g)}$ can be paired, so $\phi(e) / 2$ essentially distinct delta polynomials must be considered. Therefore, when $e<5$, the existence of delta units does not depend on the choice of $g$. For cubic fields, choosing a primitive root from the 
other class of cubic nonresidues $\mathscr{C}_{2}$ changes the signs of $\delta, c$, and the norm of the delta units.

\section{QUARTIC FIELDS}

Because we are interested in both cyclotomy and units, we will consider only the real fields, where $p \equiv 1 \bmod 8$. (The unit groups of the imaginary quartic fields are generated, up to torsion, by quadratic units.) Here we will use the normalization

$$
p=a^{2}+b^{2}, \quad b \equiv 0 \bmod 4, b>0, a \equiv 1 \bmod 4,
$$

and a primitive root $g$ is chosen (per [7]) with

$$
g^{(p-1) / 4} \equiv a / b \quad \bmod p .
$$

Theorem 2. If $K$ is a real cyclic quartic field of prime conductor $p$, the following are equivalent:

(i) $b=4$, so $K$ is a simplest quartic field as defined by Gras [3].

(ii) $K$ has a translation unit of norm +1 .

(iii) $K$ has a delta unit.

(iv) $K$ has a generalized delta unit of norm +1 .

Proof. (i) $\Rightarrow(($ ii) \& (iii)): Emma Lehmer showed that if $b=4$, then $-\eta+$ $(a-1) / 4$ is a root of the Gras quartic polynomial [3]

$$
Y^{4}-a Y^{3}-6 Y^{2}+a Y+1
$$

so it is a unit of norm +1 [10, equation (4.5), corrected]. The Lehmers later showed that if $b=4$, then either $\delta+1$ or $\delta-1$ is a unit [9], without determining which sign held for a particular $g$.

(iii) $\Rightarrow(($ iv) \& (i)): Since Hasse's [4] normalization for quartic fields agrees with ours, we will use it to obtain $\operatorname{Irr}_{\mathbb{Q}} \delta$. The symbol $\left[x_{0}, x_{1}, y_{0}, y_{1}\right]$ will represent the element of $K$ given by

$$
\left[x_{0}, x_{1}, y_{0}, y_{1}\right]=\frac{1}{4}\left(x_{0}-x_{1} \sqrt{p}+\left(y_{0}+i y_{1}\right) \tau(\chi)+\left(y_{0}-i y_{1}\right) \overline{\tau(\chi)}\right)
$$

where $\chi$ is the quartic character belonging to $K$, viz., the quartic residue symbol $\left(\frac{\cdot}{a+b i}\right)_{4}$. (Condition (4.1) is equivalent to $\chi(g)=i$ [7].) A general formula for the minimal polynomial of any element written in this way appears in [8] (or see Gras [3]). From (2.2),

$$
\delta_{0}=\eta_{0}-\eta_{1}=[-1,-1,1,0]-[-1,1,0,-1]=[0,-2,1,1] .
$$

The minimal polynomial formula now gives

$$
\operatorname{Irr}_{\mathbb{Q}} \delta=Y^{4}-p\left(Y+b^{\prime}\right)^{2}, \quad b^{\prime}=b / 4,
$$

whence

$$
N_{\mathbb{Q}}^{K}(\delta+c)=c^{4}-p\left(b^{\prime}-c\right)^{2} .
$$

Immediately we have $c=1 \Rightarrow b=4$ and norm $+1 ; c=-1$ is impossible.

(ii) $\Rightarrow$ (i): Proven in [11]. 
(iv) $\Rightarrow$ (i): From (4.3), units of norm +1 will be solutions to

$$
c^{4}-1=(c+1)(c-1)\left(c^{2}+1\right)=p\left(b^{\prime}-c\right)^{2} .
$$

There are no primes $\equiv 1 \bmod 8$ dividing the left side for $c= \pm 2, \pm 3$, and when $c= \pm 4$, the prime $p=17$ divides the left side, but $p=17$ implies $b^{\prime}=1$ and (4.4) is not satisfied. The cases $c= \pm 1$ have been handled above, so we may assume $|c| \geq 5$.

Supposing, first, that $d p=c+1$, we have $b^{\prime}=c \pm \sqrt{d(c-1)\left(c^{2}+1\right)}$. The minus root gives $b^{\prime}<0$, impossible. The plus root gives $b^{\prime}>|c|^{3 / 2}+c>$ $|c|^{3 / 2} / 4$. Then $b>|c|^{3 / 2}$, so $p>|c|^{3}$. Since $\left(b^{\prime}-c\right)^{2}>\frac{124}{125}|c|^{3}$, we are reduced to the inequality $c^{4}>\frac{124}{125} c^{6}$, which is never true for $|c| \geq 5$. The case $d p=c-1$ is virtually identical. The case $d p=c^{2}+1$ is similar. Here, $b^{\prime}=c \pm \sqrt{d\left(c^{2}-1\right)}$. Since $b^{\prime} \in \mathbb{Z}$ and $c \neq \pm 1$, we cannot have $d=1$, so the minus root is impossible. Then

$$
b^{\prime}>\frac{\sqrt{24}(\sqrt{2}-1)}{5}|c|>\frac{2|c|}{5} \Rightarrow p>\frac{64}{25} c^{2} \Rightarrow c^{4}-1=p\left(b^{\prime}-c\right)^{2}>3 c^{4},
$$

which again has no solution.

We have also proved en passant:

Corollary 4.1. A generalized delta unit of norm +1 is a delta unit with $c=1$. If $\theta=\delta \pm 1$ is a delta unit, then $b=4$, the plus sign holds, and $N_{\mathbb{Q}}^{K} \theta=1$.

Gras showed that Galois action on the roots $\theta$ of (4.2) is given by $\theta_{j+1}=$ $\left(\theta_{j}-1\right) /\left(\theta_{j}+1\right)$.

Proposition 4.2. The ordering of the $\eta$ induced by $\theta_{0}=-\eta_{0}+(a-1) / 4$ and Gras's map $\theta_{j+1}=\left(\theta_{j}-1\right) /\left(\theta_{j}+1\right)$ coincides with the ordering obtained by (2.1) and (4.1). Gras's map is the inverse of Galois action as normalized by Hasse.

Proof. The identity $\theta_{1}\left(\theta_{0}+1\right)=\theta_{0}-1$, which suffices to prove the first statement, was verified using the rule for multiplication in Hasse's basis $[4, \S 8(1)]$. Hasse normalized Galois action so that $\left[x_{0}, x_{1}, y_{0}, y_{1}\right] \rightarrow\left[x_{0},-x_{1},-y_{1}, y_{0}\right]$, and the proof of the second statement is analogous to Proposition 3.3.

Remarks. (1) Choosing a generator from the other class of nonresidues $\mathscr{C}_{3}$ changes the sign of all $\delta$, hence $c$.

(2) The only known example of a translation unit of norm -1 is $\eta-2$ in the field of conductor 401 [11]. This field does not contain a generalized delta unit. The only generalized delta unit of norm -1 which we have found is $\delta+2$ in the field of conductor 17 , which also contains delta units; no others can exist for $c^{4}+1$ squarefree.

\section{QUINTIC FIELDS}

Dickson showed [2] that the conductor $p \equiv 1 \bmod 5$ may be decomposed as

$$
16 p=x^{2}+50 u^{2}+50 v^{2}+125 u^{2},
$$

subject to

$$
x w=v^{2}-4 u v-u^{2}, \quad x \equiv 1 \bmod 5 .
$$


If $(x, u, v, w)$ is one solution to this system, the others are $(x,-v, u,-w)$, $(x, v,-u,-w)$, and $(x,-u,-v, w)$. If $g$ is a primitive root $\bmod p$, Katre and Rajwade proved in [6] that $(x, u, v, w)$ can be defined unambiguously, given $g$, by the additional condition

$$
\begin{aligned}
& g^{(p-1) / 5} \equiv(a-10 b) /(a+10 b) \bmod p, \quad a=x^{2}-125 w^{2}, \\
& b=2 x u-x v-25 v w \text {. }
\end{aligned}
$$

Conversely, if a choice of $(x, u, v, w)$ is fixed, primitive roots $g$ in only one of the four classes of quintic nonresidues in $\mathbb{Z} / p \mathbb{Z}$ will satisfy $(5.1)$. The cyclotomic numbers for such $g$ are given by

$$
\begin{gathered}
(00)=(p-14+3 x) / 25, \\
(01)=(10)=(44)=(4 p-16-3 x+50 v+25 w) / 100, \\
(02)=(20)=(33)=(4 p-16-3 x+50 u-25 w) / 100, \\
(03)=(30)=(22)=(4 p-16-3 x-50 u-25 w) / 100, \\
(04)=(40)=(11)=(4 p-16-3 x-50 v+25 w) / 100, \\
(12)=(21)=(34)=(43)=(14)=(41)=(2 p+2+x-25 w) / 50, \\
(13)=(31)=(23)=(32)=(24)=(42)=(2 p+2+x+25 w) / 50 .
\end{gathered}
$$

If we set $\delta_{j}=\eta_{j}-\eta_{j+1}$, we have, by direct computation,

$$
\begin{aligned}
\operatorname{Irr}_{\mathbb{Q}} \delta=\Delta(Y)= & Y^{5}-Y^{3} p+Y^{2} v p \\
& +\frac{p\left((3 u+v)(u-v)+5 w^{2}\right) Y}{4} \\
& +\frac{p\left(u(u-v)^{2}+(3 u-4 v) w^{2}\right)}{4} .
\end{aligned}
$$

In the quintic case, defining the periods $\eta^{\prime}$ with $g^{\prime} \in \mathscr{C}_{2}^{(g)}$ effects the substitution $(x, u, v, w) \rightarrow(x,-v, u,-w)$. Hence, the minimal polynomial of $\delta_{j}^{\prime}=\eta_{j}^{\prime}-\eta_{j+1}^{\prime}=\eta_{2 j}-\eta_{2(j+1)}$ is given by

$$
\begin{aligned}
\Delta^{\prime}(Y)= & Y^{5}-Y^{3} p+Y^{2} u p \\
& +\frac{p\left((3 v-u)(v+u)+5 w^{2}\right) Y}{4} \\
& -\frac{p\left(v(v+u)^{2}+(3 v+4 u) w^{2}\right)}{4} .
\end{aligned}
$$

The quintic analogue to a simplest field was given by Emma Lehmer in [10]. For $n \in \mathbb{Z}$ set

$$
u=n+1, \quad v=n+2, \quad w=\left(\frac{n}{5}\right)_{2},
$$

from which it follows that $x=-\left(\frac{n}{5}\right)_{2}\left(4 n^{2}+10 n+5\right)$ and

$$
p=n^{4}+5 n^{3}+15 n^{2}+25 n+25 \text {. }
$$


Lehmer showed that

$$
\theta=w \eta-\left(w-n^{2}\right) / 5
$$

is a translation unit up to sign.

The normalization $(5.1)$ of $g$ reduces to

$$
\begin{gathered}
g^{(p-1) / 5} \equiv(a-10 b) /(a+10 b) \bmod p \\
a=4\left(4 n^{4}+30 n^{2}+25\right), \quad b=-2\left(\frac{n}{5}\right)_{2}\left(2 n^{3}+20 n+25\right) .
\end{gathered}
$$

Theorem 3. Suppose $p$ is of type (5.5) and $g$ is chosen such that (5.7) holds. Then $\delta-1$ is a unit. If $p \neq 11$,

(i) $\delta-1$ is the only generalized delta unit, and

(ii) $\delta^{\prime}+c$ is never a unit.

Proof. For such $p, \Delta(Y)$ reduces to

$$
\begin{aligned}
& Y^{5}-p Y^{3}+p(n+2) Y^{2}-p n Y-p \\
& \quad=1+(Y-1)\left(Y^{4}+Y^{3}-(p-1) Y^{2}+[p(n+1)+1] Y+p+1\right) .
\end{aligned}
$$

Clearly, $\delta-1$ is a unit of norm -1 . The equations $N_{\mathbb{Q}}^{K}(\delta-c) \pm 1=\Delta(c) \pm 1=$ 0 may be considered as quintic polynomials in $c$. The lack of integer solutions to the unit equations may be proved by locating their irrational solutions between consecutive integers. If $n \geq 1$, then $\Delta(c)+1$ has a root in each open interval $(\hat{c}, \hat{c}+1)$ for

$$
\hat{c} \in\left\{-n^{2}-3 n-6,-1,0, n+1, n^{2}+2 n+3\right\} .
$$

In each case, $\operatorname{sgn}(\Delta(\hat{c})+1) \neq \operatorname{sgn}(\Delta(\hat{c}+1)+1)$. This accounts for all five roots, so there are no generalized delta units when $n \geq 1$. The polynomial $\Delta(c)-1$ has an exact root at $c=1$ instead of an irrational root in $(0,1)$; otherwise, its four irrational roots are located in the same intervals. Similar results hold for $n<-3$. The case $n=-3$ yields no solutions for $c$, which leaves only $p=11$. Hence (i). For the proof of (ii), replace $\Delta$ by $\Delta^{\prime}$ and proceed in the same way.

Corollary 5.1. Take $x, u, v, w, p, a$, and $b$ as above and define the periods with an arbitrary primitive root $g$. If $p=11$, all $g$ define an ordering such that $\Delta(Y)$ has delta units. Otherwise, $\Delta(Y)$ has delta units if and only if $g$ satisfies

$$
g^{(p-1) / 5} \equiv\left(\frac{a-10 b}{a+10 b}\right)^{ \pm 1} \bmod p .
$$

These are the $g$ in two (i.e., half) of the four nonresidue classes.

Proof. This is immediate from the theorem and (5.1).

The field of conductor 11 is a special case. It is of type (5.5) with either $n=-2$ or $n=-1$. (One can show that 11 is the only integer represented 
nonuniquely by the polynomial (5.5).) The period polynomial for $p=11$ is

$$
Y^{5}+Y^{4}-4 Y^{3}-3 Y^{2}+3 Y+1
$$

so the periods $\eta$ are themselves units. Also $\eta \pm 1$ and $\eta+2$ are Galoisconjugate units (but not conjugate to $\eta$ ). Choosing to use $n=-2$, we have from (5.3) and (5.4) that $\delta-1, \delta+2, \delta-3, \delta^{\prime} \pm 1$, and $\delta^{\prime}+2$ are all units, no two conjugate.

The converse of Theorem 3 is false. In the field of conductor 211 using $(x, u, v, w)=(1,1,2,-5), \delta-1$ is a unit of norm -1 . There is a generalized delta unit $\delta-3$ for $p=61$ and $(x, u, v, w)=(1,1,4,-1)$.

Schoof and Washington showed that Galois action on the quintic translation units (5.6) can be given by

$$
\theta \rightarrow \frac{(n+2)+n \theta-\theta^{2}}{1+(n+2) \theta} .
$$

When $g$ satisfies (5.7), then (5.6) induces an ordering of the $\theta_{j}$. The method of Proposition 3.2 can be used to show that with this ordering the image of $\theta_{0}$ under (5.8) is $\theta_{2}$ when $w=1$, and $\theta_{3}$ when $w=-1$. In [11], the map (5.8) was derived from (5.6) and the canonical ordering of the $\eta_{j}$, but we have changed the normalization of $(x, u, v, w)$ from [10] and [11]. The normalizations (3.1), (4.1), and (5.1) all follow naturally from Jacobi sums; they insure that the character defined by $\chi(g)=\zeta_{e}$ coincides with the particular $e$ th-power residue symbol modulo $p$ belonging to the field $K$ [5]. Using Lehmer's $u$ and $v$ with normalized $g$ makes the units translates of $\delta^{\prime}$ instead of $\delta$. Changing $u$ and $v$ seemed the lesser evil.

Remark. We were unable to find any infinite family of quintic fields with generalized delta units containing either $p=61$ or $p=211$. Furthermore, we were unable to make any progress on the conjecture of Schoof and Washington in [11] that all quintic fields with translation units are of Emma Lehmer's form (5.5).

\section{BIBLIOGRAPHY}

1. Paul Bachmann, Die Lehre von der Kreisteilung, Teubner, Leipzig and Berlin, 1927.

2. Leonard E. Dickson, Cyclotomy, higher congruences, and Waring's problem, Amer. J. Math. 57 (1935), 391-424.

3. Marie-Nicole Gras, Table numérique du nombre de classes et des unités des extensions cycliques de degré 4 de $\mathbb{Q}$, Publ. Math. Fasc. 2, Fac. Sci. Besançon, 1977/1978.

4. Helmut Hasse, Arithmetische Bestimmung von Grundeinheit und Klassenzahl in zyklischen kubischen und biquadratischen Zahlkörpern, Math. Abhandlungen, vol. 3, Walter deGruyter, Berlin, 1975, pp. 285-379; originally published 1950.

5. S. A. Katre and A. R. Rajwade, Complete solution of the cyclotomic problem in $F_{q}$ for any' prime modulus $l, q=p^{\alpha}, p \equiv 1(\bmod l)$. Acta Arith. 45 (1985), 183-199.

6. __ Unique determination of cyclotomic numbers of order five, Manuscripta Math. 53 (1985), 65-75.

7. Resolution of the sign ambiguity in the determination of the cyclotomic numbers of order 4 and the corresponding Jacobsthal sum, Math. Scand. 60 (1987), 52-62.

8. Andrew J. Lazarus, Gaussian periods and units in certain cyclic fields, Proc. Amer. Math. Soc. 115 (1992), 961-968. 
9. D. H. Lehmer and Emma Lehmer, The Lehmer project, Math. Comp. 61 (1993), 313-317.

10. Emma Lehmer, Connection between Gaussian periods and cyclic units, Math. Comp. 50 (1988), 535-541.

11. René Schoof and Lawrence C. Washington, Quintic polynomials and real cyclotomic fields with large class numbers, Math. Comp. 50 (1988), 543-556.

12. Daniel Shanks, The simplest cubic fields, Math. Comp. 28 (1974), 1137-1152.

13. Thomas Storer, Cyclotomy and difference sets, Markham, Chicago, 1967.

Department of Mathematics, University of California, Davis, California 95616

Current address: 2745 Elmwood Avenue, Berkeley, California 94705 